3 Heim C, Newport DJ, Mletzko T, Miller AH, Nemeroff CB. The link between childhood trauma and depression: insights from HPA axis studies in humans. Psychoneuroendocrinology 2008; 33: 693-710.

4 Boyce $\mathrm{P}$, Mason C. An overview of depression-prone personality traits and the role of interpersonal sensitivity. Aust N Z J Psychiatry 1996; 30: 90-103.

Hans G. Stampfer, Professor of Psychiatry, University of Western Australia, Australia. Email: hans.stampfer@uwa.edu.au

doi: 10.1192/bjp.202.4.310b

Authors' reply: We would like to clarify a few points regarding our conclusion that our results 'strengthen the theory of a cardiovascular contribution to the aetiology of depression.'

First, we talk about a 'contribution' which does not necessarily imply a direct causal pathway, stating that 'our findings are not explanatory with respect to causal chains leading to the onset of depression'. In line with this, we did include a careful discussion about other possible confounding mechanisms, i.e. factors that may increase the risk for both poor fitness and depression - for example, childhood factors, personality, self-esteem and subsyndromal affective problems. By including parental educational level as a confounder and by performing subanalyses within full brother pairs, many of the early childhood risk factors could be accounted for.

Second, as the conscription routines included extensive questions regarding every possible previous and present mental health problem in combination with separate examinations by professional psychologists and physicians, we believe that subsyndromal affective problems were not often overlooked. Also, to further reduce baseline misclassification, we did perform separate analyses excluding incident cases in the first year.

Third, we would like to stress that not all study participants were fit for recruitment into national service, but that the conscription test was used to select suitable recruits. Participation in the conscription tests was compulsory according to Swedish law and exemptions were granted only for incarcerated males and severe chronic medical disabilities (approximately 2-3\% of the yearly male population). We can therefore consider our study a population study. After conscription, about 40000 individuals were considered 'unfit' due to a cardiovascular fitness stanine score 1-3. All these 'unfit' young men were included in our study.

Fourth, the question of whether cardiovascular fitness may be related to increased risk for other types of psychiatric disorders in adulthood is one that we will continue to pursue in future analyses of the national conscription data.

Taken together, we still argue that the data 'strengthen the theory of a cardiovascular contribution to the aetiology of depression', which in our paper stands in direct connection with the sentence: 'although the results in the present population-based prospective study are compelling, a number of confounders could not be measured and intervention studies are needed to determine whether physical exercise in young adulthood can prevent future onset of depression.' We fully agree with de Jonge \& Roest in their editorial $^{2}$ that a greater understanding of the mechanisms underlying these associations, including complex bidirectional models, may provide opportunities and strategies for prevention.

1 Åberg MAl, Waern M, Nyberg J, Pedersen NL, Bergh Y, Åberg DN, et al. Cardiovascular fitness in males at age 18 and risk of serious depression in adulthood: Swedish prospective population-based study. Br J Psychiatry 2012; 201: 352-9.

2 de Jonge $\mathrm{P}$, Roest AM. Depression and cardiovascular disease: the end of simple models. Br J Psychiatry 2012; 201: 337-8.
H. Georg Kuhn, Institute for Neuroscience and Physiology, University of Gothenburg, Medicinaregatan 11, Box 436 (CBR), S-40530 Gothenburg, Sweden. Email: Georg.Kuhn@neuro.gu.se; Maria A. I. Aberg, Center for Brain Repair and Rehabilitation, Institute for Neuroscience and Physiology and Department of Primary Health Care, Institute of Medicine, University of Gothenburg, Sweden; Margda Waern, Department of Psychiatry and Neurochemistry, Neuropsychiatric Epidemiology Unit, Institute of Neuroscience and Physiology, University of Gothenburg, Sweden; Jenny Nyberg, Michael Nilsson, Center for Brain Repair and Rehabilitation, Institute for Neuroscience and Physiology, University of Gothenburg, Sweden; Nancy L. Pedersen, Department of Psychology, University of Southern California, LOS Angeles, California, USA, and Department of Medical Epidemiology and Biostatistics at Karolinska Institute, Stockholm, Sweden; Ylva Bergh, Department of Primary Health Care, Institute of Medicine, University of Gothenburg, Sweden; $\mathbf{N}$. David Åberg, Laboratory of Experimental Endocrinology, Institute of Medicine, University of Gothenburg, Sweden; Kjell Torén, Occupational and Environmental Medicine, Institute of Medicine, University of Gothenburg, Sweden

doi: 10.1192/bjp.202.4.311

\section{New paradigm: developmental psychopathology}

Strong on diagnosis, but weak on prescription, Bracken et al's ${ }^{1}$ critique of contemporary psychiatry suffers from the very difficulty which they decry. They rightly complain that current paradigms ignore the psychosocial, fail to combat stigma, and that academic psychiatry has little impact on clinical practice. They cogently argue that the relational aspects of treatment, whether avowedly psychotherapeutic or pharmacological, outweigh any supposed specificity in their effectiveness.

Sadly, their remedies are vague and anodyne: encouraging service user involvement, acknowledgement of complexity, taking account of 'systems of meaning'. Motherhood and apple pie anyone? This anti-psychiatry rehash sounds the retreat rather well, but as a call to arms is feeble; it knows what it is 'anti', but lacks a convincing 'pro'.

Yet there is in fact an exciting way forward, one where academic psychiatry and psychology convincingly combine to enhance work in the clinic. Developmental psychopathology is the current cutting edge, drawing on attachment theory, neuroimagining and epigenetics. ${ }^{2}$ We are beginning to see how developmental experience inscribes itself on the brain, and sometimes on the genome; how the interaction of adverse developmental processes within the social milieu sows the seeds for psychiatric disorder. This provides the intellectual and evidential underpinning for effective psychotherapeutic treatments, which enhance resilience through fostering mentalising and mindfulness skills, promoting a sense of agency, and validating appropriate help-seeking. Psychiatrists-of-the-future's enthusiasm needs to be fanned by this flowering of environmental neuroscience, rather than doused with thin foam of post-modern angst.

1 Bracken P, Thomas P, Timimi S, Asen E, Behr G, Beuster C, et al. Psychiatry beyond the current paradigm. Br J Psychiatry 2012; 201: 430-4.

2 Holmes J. Psychodynamic psychiatry's green shoots. Br J Psychiatry 2012: 200: 439-41.

Jeremy A. Holmes, Visiting Professor, University of Exeter, UK. Email: j.a.holmes@btinternet.com

doi: 10.1192/bjp.202.4.311a

Territorial disputes are a zero sum game: if one side gains ground, it can only be at the expense of the other. As clinical psychologists, it was therefore with a wry smile that we read the recent paper by Bracken and colleagues, ${ }^{1}$ which calls for psychiatry 'to move beyond the dominance of the current, technological paradigm' and towards an understanding of mental health problems not as 